\title{
Integrating the map and the territory
}

"V

ertical integration" is all the rage in Canadian health care reform these days: a struggle is underway to get hospitals and primary care providers communicating, coordinating and cooperating. Improved health outcomes and a better patient experience are the main goals, but other benefits include fewer avoidable hospital admissions, shorter emergency department wait times, and ultimately, a leveling off of health care spending.

Integrated health systems may well be able to achieve all this - and more.' Imagine a system in which family doctors know when their patients are admitted and discharged from hospital and what happened while they were there; patients at high risk of admission receive more intensive care in the community; and most of all, care providers actually talk to each other, sharing advice and information in the best interests of the patient.

Getting there is about integrating not only systems, but perspectives. It is about understanding the difference between what Dr. Ian McWhinney called (borrowing from Korzybski's metaphor) "the map" and "the territory." The map is the birds-eye view: it allows us to see the populations we need to focus on, the gaps in care and the effects of programs instituted to address them. It is a perspective traditionally held by system thinkers and administrators rather than clinicians, and it is a vital one.

The territory is the individual's lived experience of illness. As McWhinney put it, "if we are to be healers as well as technicians, we have at some point to set aside our maps and walk hand-inhand with our patients through [it]." This is where good family doctors shine, and where our specialist and system partners can often learn from us.

McWhinney, the extraordinary physician from the University of Western Ontario, often referred to as the Father of Family Medicine, died Sept. 28, 2012. One of his many gifts was his ability to distill and articulate the culture of general/family practice; to describe the lived

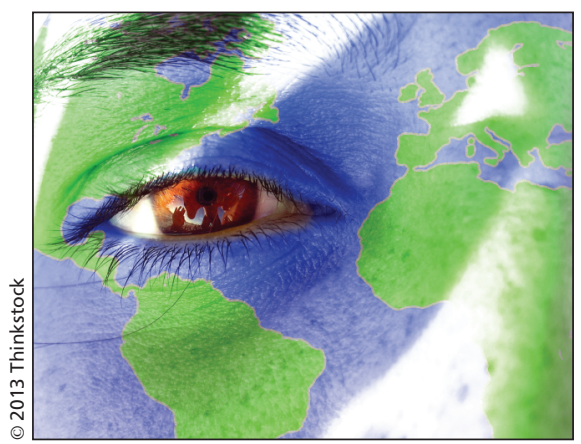

experience of talented clinicians who had until then not really thought of their work as being worthy of study or analysis.

In a famous 1996 lecture, The Importance of Being Different,${ }^{2}$ McWhinney outlined the four major ways general practice differs from other specialties. The first two are particularly relevant to the challenge of vertical integration:

- [Family practice] is the only discipline to define itself in terms of relationships, especially the doctorpatient relationship.

- General practitioners tend to think in terms of individual patients, rather than generalized abstractions.

Some specialists will bristle at the first claim, arguing that they too develop relationships with their patients. But as McWhinney points out, in family medicine "the relationship is usually prior to content. We know people before we know what their illnesses will be." This relationship focus has implications for how family doctors respond to system reforms and how we interact with our colleagues in the system.

The second claim may seem perplexing given that pattern recognition is the basis of diagnosis, and evidencebased medicine sometimes requires treatment by algorithm. But patients are people embedded in complex biological, personal and social contexts ${ }^{3}-$ and much more of that complexity is visible in primary care than in hospital.

This reality has positive and negative consequences. Family doctors have sometimes pushed back against reforms they perceive as undermining their rela- tionships with patients (like specialty clinics that pull patients out of their care for one particular disease or body part) or "cookie-cutter" approaches to disease management (like practice guidelines that they perceive as inflexible). Furthermore, our discipline sometimes struggles to maintain the relationship focus within new models of care that can be patient centred but less focused on the doctor-patient dyad.

Some reforms must be pushed in spite of resistance, but we mustn't forget the very robust body of evidence indicating that a well-functioning primary care system is the best way to improve health outcomes and reduce system costs. ${ }^{4}$ So the territory-level family medicine approach is on to something.

Indeed, many well-intentioned reforms (including clinical practice guidelines) have faltered in Canada because of an obsession with the map and the absence of any focus on the territory. This could often have been addressed by involving family doctors from the outset.

When administrators and specialists sit down with primary care providers to work with us to integrate services, they will be met with a tidal wave of stories: which patients have been well-served, which ones ill-served and why. This is not evidence by anecdote. It is narrative. It is the territory. Its importance may not be immediately obvious to those whose perspective is at the level of the map. If it is recognized as a central driving force of family medicine, and if it is given its due, integration will have some hope of succeeding. If it is written off as microlevel thinking, we are all sunk.

\section{Danielle Martin MD \\ Department of Family and \\ Community Medicine \\ Women's College Hospital \\ University of Toronto \\ Toronto, Ont.}

For references, see Appendix 1, available at www.cmaj.ca/lookup/suppl/doi:10.1503 /cmaj.12759/-/DC1

CMAJ 2013. DOI:10.1503/cmaj.121759 